\title{
A WAVELET REPRESENTATION OF HVSR TECHNIQUE
}

\author{
G. Hloupis ${ }^{1,2}$, F. Vallianatos ${ }^{1}$ and J. Stonham ${ }^{2}$ \\ ${ }^{1}$ Department of Natural Resources \& Environment, Technological Educational Institute of Crete, \\ Romanou 3, 73133 Chania, GREECE, fvallian@chania.teicrete.gr \\ ${ }^{2}$ Department of Electronic and Computer Engineering, Brunel University, Uxbridge, Middlesex \\ UB83PH, UNITED KINGDOM, george.hloupis@brunel.ac.uk, john.stonham@brunel.ac.uk
}

\begin{abstract}
In order to evaluate eathquake site amplification characteristics, horizontal to vertical Fourier spectrum of microtremor has been widely used. As long as the Fourier transform (FT) cannot distinguish between stationary and non-stationary coefficients we cannot eliminate the contamination of microtremors signals from short period transients.

The wavelet transform (WT), using the property of localization of wavelet bases has been widely used in signal processing. Unlike the Short Time Fourier Transform (STFT) in which the width of window is fixed, the WT localizes signal in a variable window using the dilation parameter. This property, which derived directly from multiresolution analysis provide us the ability to decompose a signal in a well localized set of coefficients and identify the non-stationary portions of it.

In the present study we use the WT in order to eliminate the non-stationarities in microtremor signals before we calculate the spectrum of each one using conventional FFT algorithms
\end{abstract}

\section{INTRODUCTION}

With the term "microtremors" we define Earth's surface vibrations caused by daily human activities such as movement of machinery in factories, motor cars, people walking and natural phenomena such as flow of water in rivers, rain, wind, variation in atmospheric pressure and ocean waves.

The spectral ratio between horizontal and vertical components ( $\mathrm{H} / \mathrm{V}$ ratio) of microtremors (as measured from triaxial seismometers at the ground surface) has been used to estimate fundamental periods and amplification factors of a site in order to identify the site's response during earthquake. This technique was first applied by Nogoshi and Igarashi $(1970,1971)$ and popularized by Nakamura (1989) in order to determine the predominant frequency and amplification of a site. The preciseness of this method depends on the stationarity of the recorded microtremor signals. Nonstationary components (such as transients) contaminate the signal and reveals frequencies that are not related with site's characteristics.

In the present study we will estimate first the Horizontal to Vertical Spectrum Ratio (HVSR) using ordinary methodology and then we calculate the HVSR using wavelet-filtered microtremor signals in order to identify the variation of HVSR when the non-stationary components elimininated.

\section{SIGNAL ANALYSIS}

\subsection{Conventional Methods}

The Fourier analysis (Papoulis, 1962), which decomposes a time series into orthogonal frequency components or vice versa, has been widely used for geophysical signal processing. The fast Fourier transform (FFT) algorithm has made the Fourier analysis very attractive for many data processing applications, mainly because of the orthogonal properties of the Fourier series and of its simple expression. However, upon inspection of the expression of FT 


$$
F(\omega)=\int_{-\infty}^{\infty} f(t) e^{-i \omega t} d t
$$

one must notice that the FT extracts periodic signals from a function $f(t)$ of infinite time duration and the frequency information cannot be associated with time. One can only analyze and process signals in either the time or the frequency domain.

To compensate for the limitations of the FT related with time-frequency analysis, the concept of the short time Fourier transform (STFT) was developed. This transform which also called Gabor transform is defined as (Chui, 1992)

$$
G_{k, b}(\omega)=\int_{-\infty}^{\infty} f(t) e^{-i \omega t} g_{k}(t-b) d t
$$

where $g(t)$ is a Gaussian function

$$
g_{k}(t)=\frac{1}{2 \sqrt{\pi \cdot k}} e^{-\frac{t^{2}}{4 k}}
$$

which also called a window function. (Alternative formulations replaced the Gaussian function by other window functions). Thus the Gabor transform localizes the FT of a signal $f(t)$ at around $t=b$. The width of the window is determined by the fixed positive constant k. In the majority of STFT methods there are undesirable computational complexities when either narrowing of the window is required for better localization or widening the window required to obtain more global picture (Chui, 1992).

\subsection{Wavelet transform (WT)}

Wavelets are functions that satisfy certain mathematical requirements and are used in representing data or other functions. The class of functions that present the WT are those that are square integrable and satisfy that when the frequency approaches zero, the Fourier transform of the wavelet function must also approach zero. This class of functions is denoted as:

$$
f(x) \in L^{2}(R) \Rightarrow \int_{-\infty}^{+\infty}|f(x)|^{2} d x<\infty \text {. }
$$

The wavelet functions that used in the wavelet analysis are derived from a single prototype function (the mother wavelet) by dilating (scaling) and translating (time shifting) its two characteristic parameters, the dilation (a) and the translation (b), which vary continuously. A wavelet function $\psi_{a, b}(x)$ can be defined as

$$
\psi_{a, b}(x)=\frac{1}{\sqrt{|a|}} \psi\left(\frac{x-b}{a}\right) \quad a, b \in R \text { with } a \neq 0
$$

The parameter a (dilation) controls the number of dilations hence it provides the frequency resolution of the wavelet function as long as the parameter $b$ (translation) controls the position of wavelet in time.

Whereas the Fourier transform decomposes a signal into infinite length sines and cosines, effectively losing all time-localization information, the CWT's basis functions are scaled and shifted versions of the time-localized mother wavelet. The CWT is a convolution of the data sequence with a scaled and translated version of the mother wavelet, the $\psi$ function such as

$$
\left(W_{\psi} f\right)(a, b)=\left\langle f, \psi_{(\alpha, b)}\right\rangle=\frac{1}{\sqrt{|a|}} \int_{-\infty}^{\infty} f(t) \psi\left(\frac{t-b}{a}\right) d t .
$$
scale.

Equation (6) contains normalization so that the wavelet function contains unit energy at every

In the CWT, for each value of the scale used, the correlation between the scaled wavelet and successive segments of the data stream is computed. Unless reconstruction is needed, there are 
no restrictions in the CWT as to how many scales are used, nor of the spacing between the scales. The convolutions can be done up to $\mathrm{N}$ times at each scale, and must be done all $\mathrm{N}$ times if the FFT is used. The CWT consists of $\mathrm{N}$ spectral values for each scale used, each of these requiring an inverse FFT. The computational load of the CWT and its memory requirements are thus considerable. The benefit from this high measure of redundancy in the CWT is an accurate time-frequency spectrum.

The selection of the mother wavelet function is critical for the results that we expect to derive. In our analysis we choose one of the most known and widely used in geophysical signals is the Morlet wavelet. This wavelet consists of a plane wave modulated by Gaussian function and is defined as:

$$
\psi_{0}(\eta)=\pi^{-\frac{1}{4}} e^{i d_{1} \eta} e^{-\frac{\eta^{2}}{2}}
$$

$d_{o}$ is the so-called "wavenumber" of the wavelet and is adjustable from 6 (minimum value to satisfy the admissibility condition) to 500 , where small values provides better time resolution against frequency resolution and vice versa. In Figure 1 we have a morlet wavelet with wavenumber 6 .

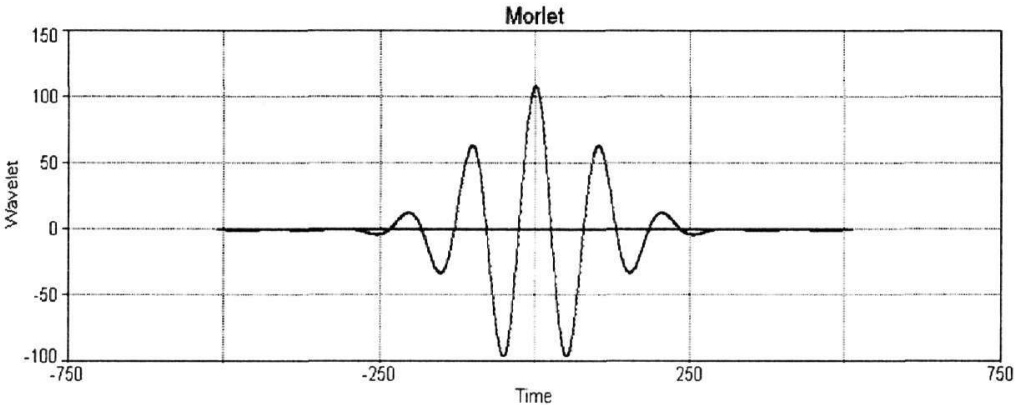

Figure 1. Morlet wavelet $\left(d_{0}=6\right)$.

In practice the CWT is interpreted as the signal $f$ is filtered by a particular (non-uniforms, bandpass) filter banks $\psi$. These filters decompose the signal's spectrum into sub-spectra of different bandwidths (wavelet's wavenumber). Octave filter banks (filters with exponentially spaced centre frequency and bandwidths) are used in order to perform the WT (Mallat, 1989). Decomposition algorithm exists to compute scaling function and wavelet coefficients at level $j$ from the scaling function at level $j-1$ (Odgen, 1997). This algorithm implemented as a pair of filtering operations. The decomposition scheme builds from two-channel tree-stucture filter banks consisting of a low-pass filter $\mathrm{H}$ and a high pass filter $\mathrm{G}$ as shown in Figure 2, for $\mathrm{a} j=3$.

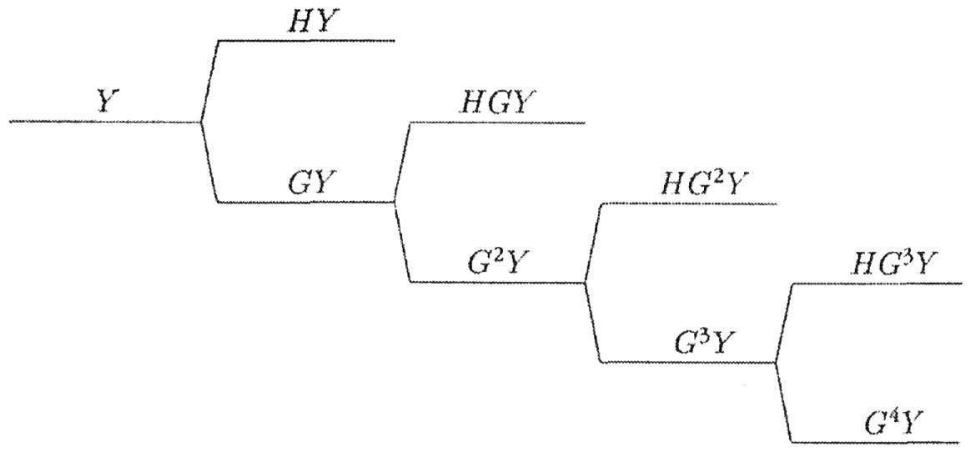

Figure 2. Tree diagram for $\mathrm{j}=3$ wavelet decomposition algorithm.

The plot of the coefficients of WT is called scalogram. Usually it consists of the square of the CWT (or another parameter that can be calculated from wavelet coefficients) which plotted against scale and translation over time. 
Just as it is possible to reconstruct a signal $f(t)$ given its Fourier transform $F(\omega)$ by means of the inverse Fourier transform, it is also possible to reconstruct a signal given only its continuous wavelet transform $\left(\mathrm{W}_{\psi} f\right)(\alpha, b)$. For an appropriate wavelet $\psi$ and for $f \in L^{2}(\Re)$

$$
f(t)=\frac{2}{C_{\psi}} \int_{-\infty}^{\infty} \int_{0}^{\infty}\left(W_{\psi} f\right)(\alpha, b) \psi_{(\alpha, b)}(t) \frac{1}{\alpha^{2}} d a d b
$$

for every $t \in \mathfrak{R}$ at which $\mathrm{f}$ is continuous. The constant $\mathrm{C}_{\psi}$ is given by

$$
C_{\psi /}=2 \int_{0}^{\infty} \frac{\left.\hat{\psi}(\omega)\right|^{2}}{\omega} d \omega=2 \int_{0}^{\infty} \frac{|\hat{\psi}(-\omega)|^{2}}{\omega} d \omega
$$

\section{ESTIMATION OF HVSR}

The horizontal to vertical spectral ratio (HVSR) is estimated from the three components of the microtremor recordings (North-South, East-West and Vertical) through the following steps (Magri et al.1994, Mucciarelli 1998) :

a) Offset removal: the mean of the entire recording is deducted from each sample.

b) Single window processing: The second step consists in selecting portions of the signal that do not contain transients. This is done manually (by visual examination of the recording) or with the aid of automatic window selection algorithms based on well-known STA/LTA approaches.

c) Process of individual windows:

- Bandpass filtering (usually from 0.1 to $20 \mathrm{~Hz}$ )

- Cosine tapering with a length of $5 \%$ is applied on both side of the window signal of the vertical, North-South and East-West component.

- FFT is applied to each component in order to obtain the spectral amplitudes.

- A smoothing function is applied (usually Konno \& Ohmachi window function) to each spectral amplitude (Kohno et al. 1995).

- Merging of two horizontal spectral amplitudes with a quadratic mean,

$$
H=\sqrt{\frac{N S^{2}+E W^{2}}{2}}
$$

Thus in each of the windows the distribution of $\log _{10}(\mathrm{H} / \mathrm{V})$ is obtained as a function of frequency.

d) Average results

$\mathrm{H} / \mathrm{V}$ is averaged over all window results

\section{APPLICATION TO MICROTREMORS}

\subsection{Data acquisition}

Microtremors recordings were collected with Reftek DAS- 130 data logger at $125 \mathrm{~Hz}$ using Sercel's L3-4D seismometer at Chania, Greece. One set of three microtremor recordings is presented below (Fig. 3a, 3b, 3c) 


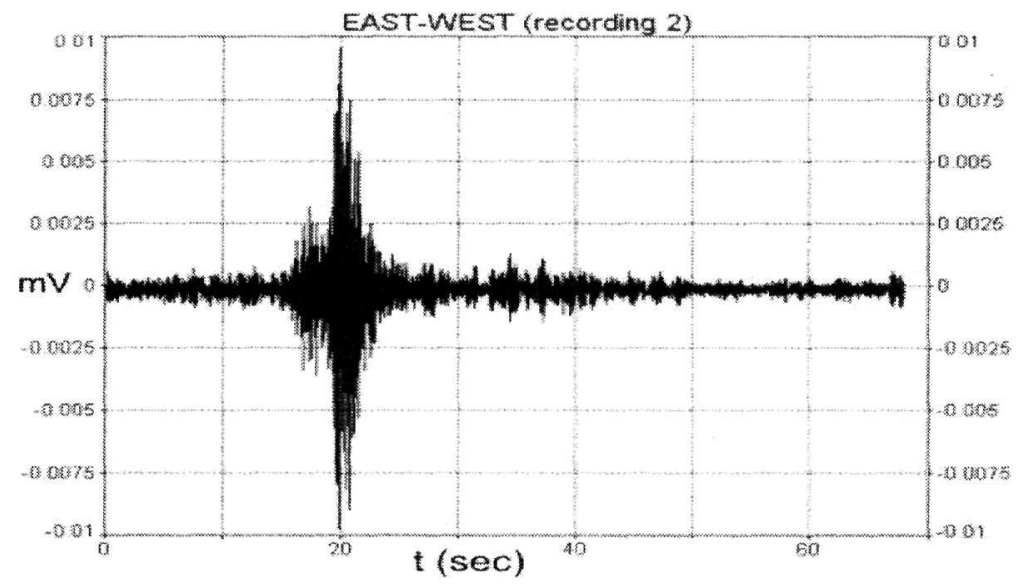

Figure 3a. Horizontal (East-West) component

NORTH-SOUTH (recording 2)

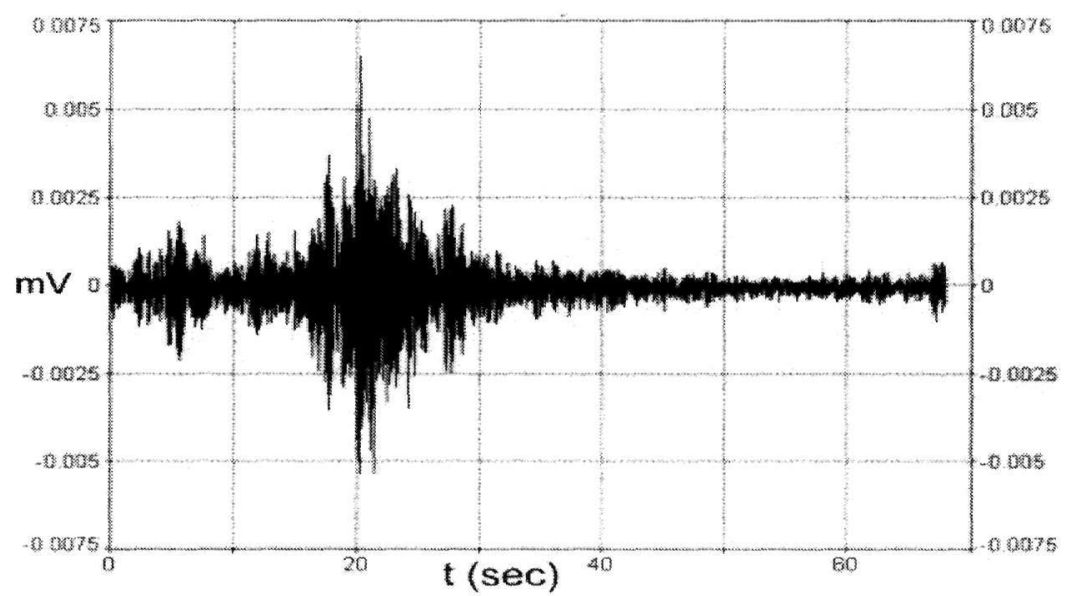

Figure 3b. Horizontal (North-south) component

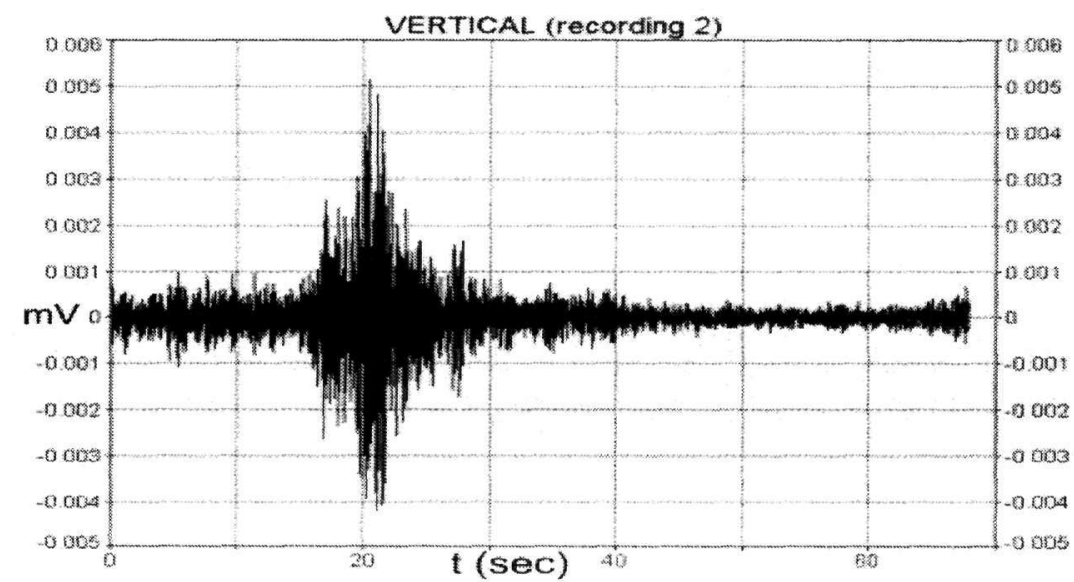

Figure 3c. Vertical component 


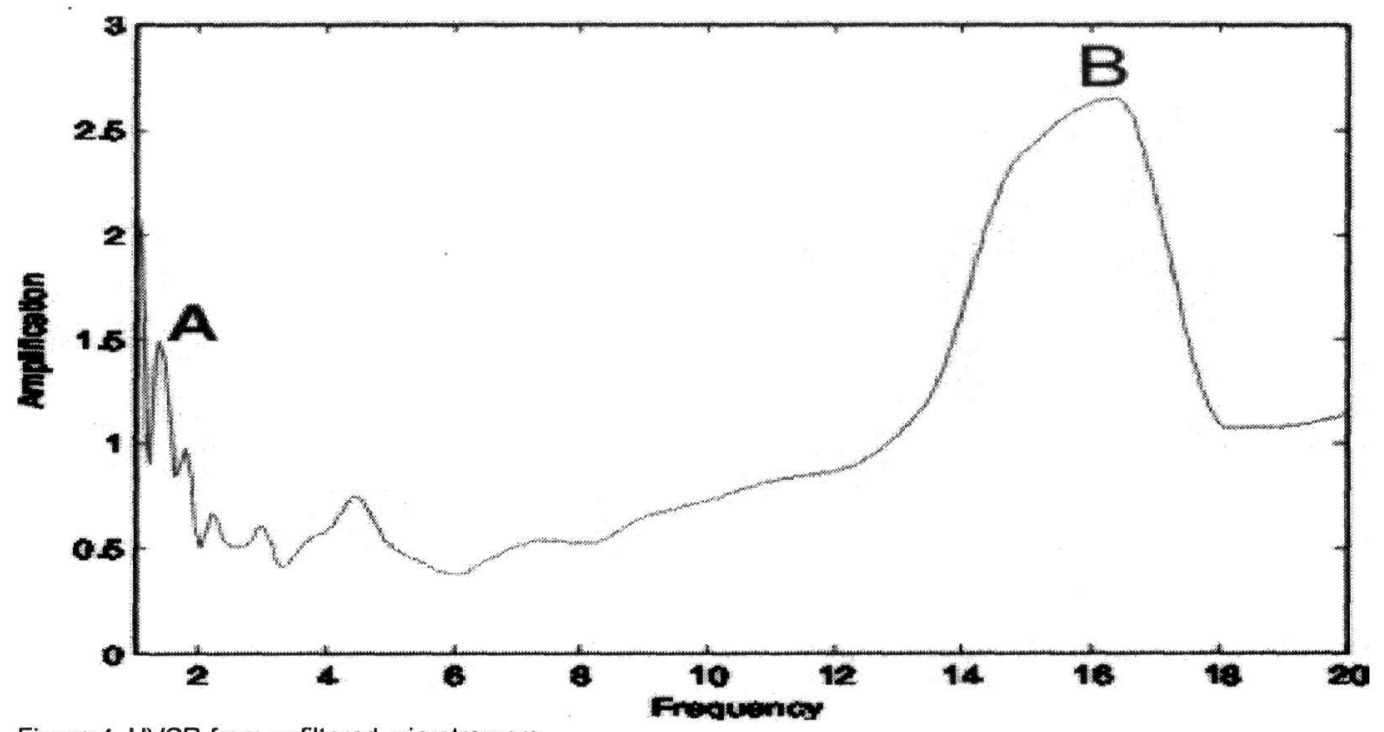

Figure 4. HVSR from unfiltered microtremors

\subsection{Using WT and filtering in HVSR estimation}

We estimate the HVSR following the steps defined in section 3. The calculated amplification factor $S$ over frequency is depicted in Figure 4. We can identify amplification for frequencies around $1.1 \mathrm{~Hz}$ (point A) and around $16.4 \mathrm{~Hz}$ (point $\mathrm{B}$ )

As mentioned earlier HVSR method needs stationary data because any non-stationary event (transients, spikes e.t.c) contaminates the spectrum ratio because its frequency components take place in the calculation of spectral ratio. For this reason we altered the steps that presented in section 3 and specifically we split the step "B" with the following two:

b1) Calculation of Wavelet spectrum using (5) or (6)

b2) Wavelet filtering for time and frequency simultaneously.

From the b1) we can identify clearly the non-stationary components of the microtremor gathering both time and frequency information. The calculated wavelet spectrum of recorded microtremors are depicted in Figures $5 \mathrm{a}, 5 \mathrm{~b}$ and $5 \mathrm{c}$.

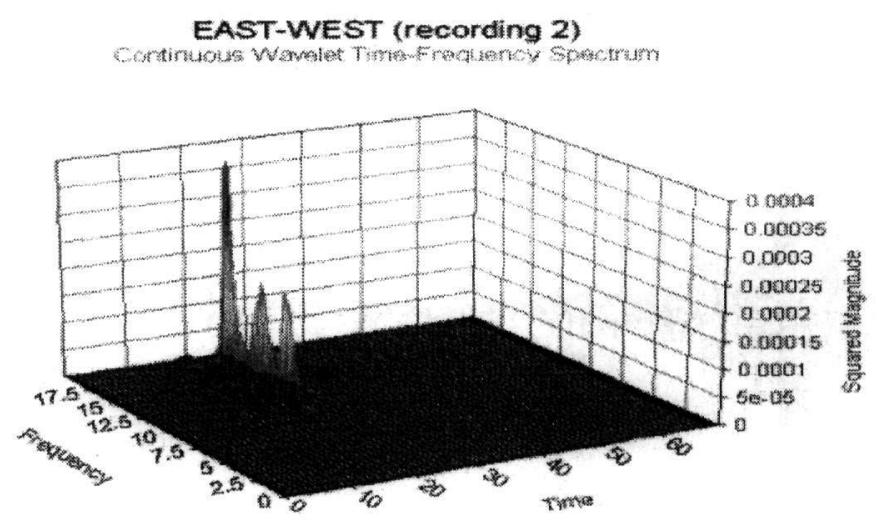

Figure 5a. Wavelet spectrum for E-W component 


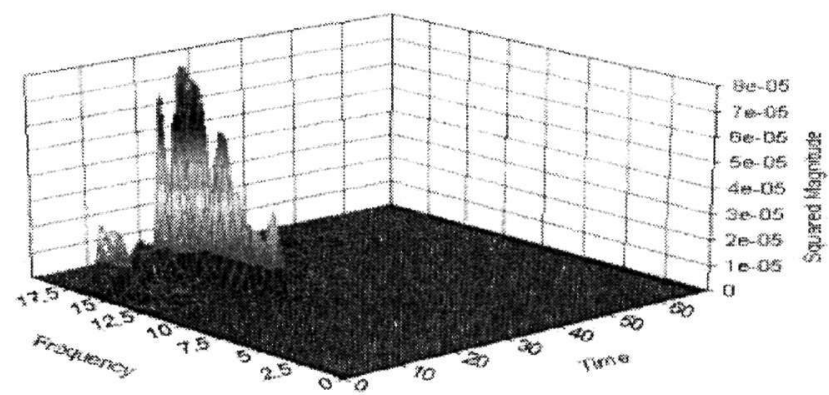

Figure $5 b$. Wavelet spectrum for $\mathrm{N}-\mathrm{S}$ component

VERTICAL (recording 2)

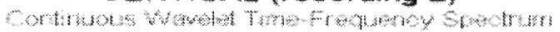

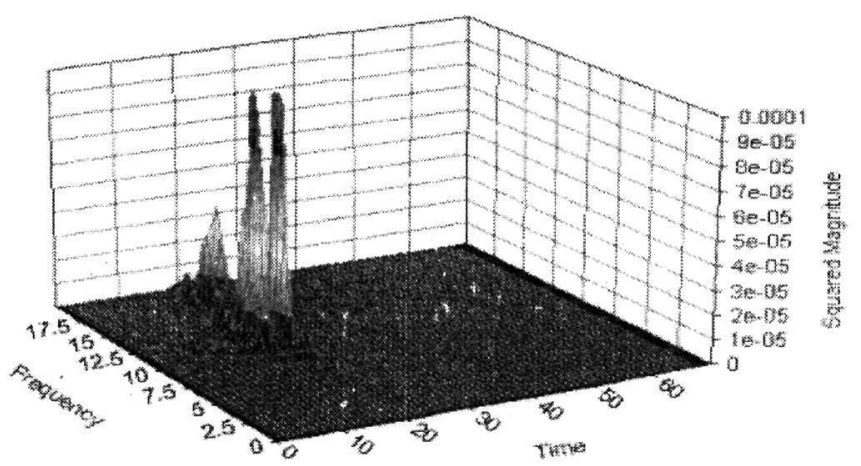

Figure $5 \mathrm{c}$. Wavelet spectrum for Vertical component

From the above figures we can clearly identify non-stationary components with frequencies from $8 \mathrm{~Hz}$ to $18 \mathrm{~Hz}$ between $15 \mathrm{sec}$ and $25 \mathrm{sec}$. From this point as long as we identify the undesired portions of the microtremor we apply a wavelet cut-off filter (non-linear filter which eliminates signal's components using both time and frequency parameters). The filter that we apply blocked the frequencies from 8 to $18 \mathrm{~Hz}$ for 15 to $25 \mathrm{sec}$ time interval as depicted in microtremor recording shown in Figures $6 a, 6 b$ and $6 c$.

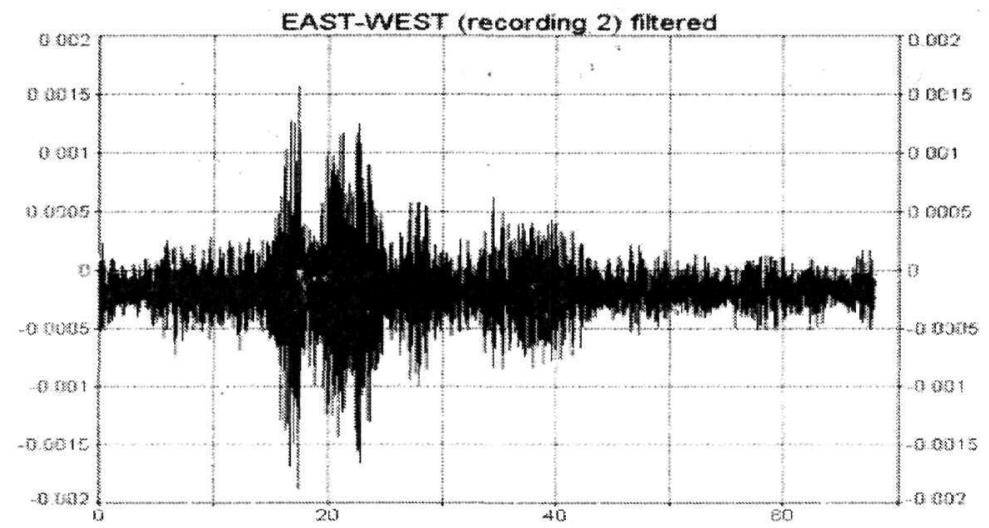

Figure 6a. Horizontal (East-West) component filtered 


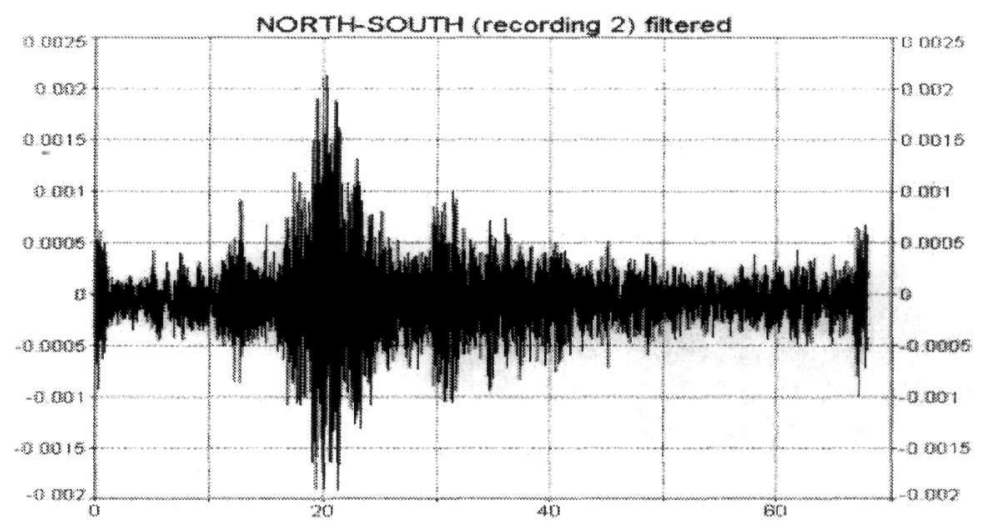

Figure 6b. Horizontal (North-South) component filtered

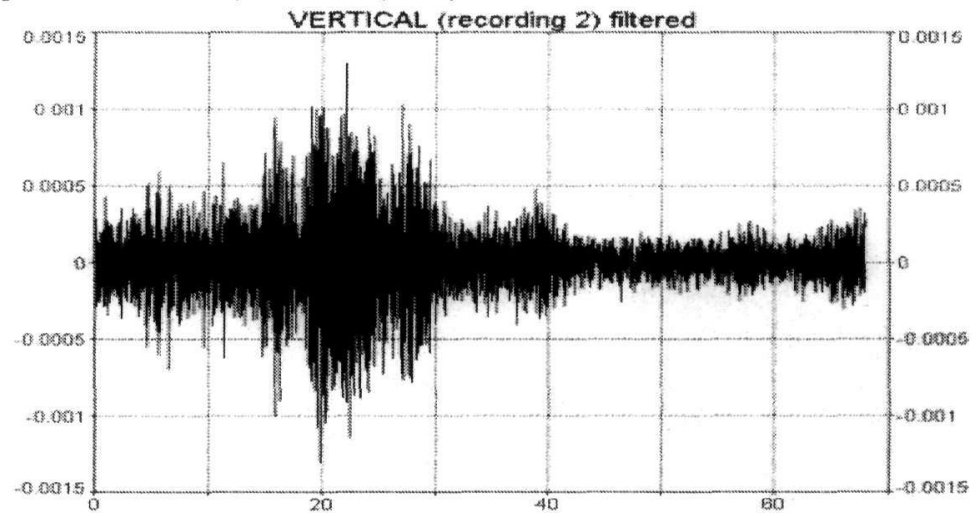

Figure 6c. Vertical component filtered

For evaluation of filtering correctness we present below the calculated wavelet spectrums after filtering (Fig $7 \mathrm{a}, 7 \mathrm{~b}$ and $7 \mathrm{c}$ ).

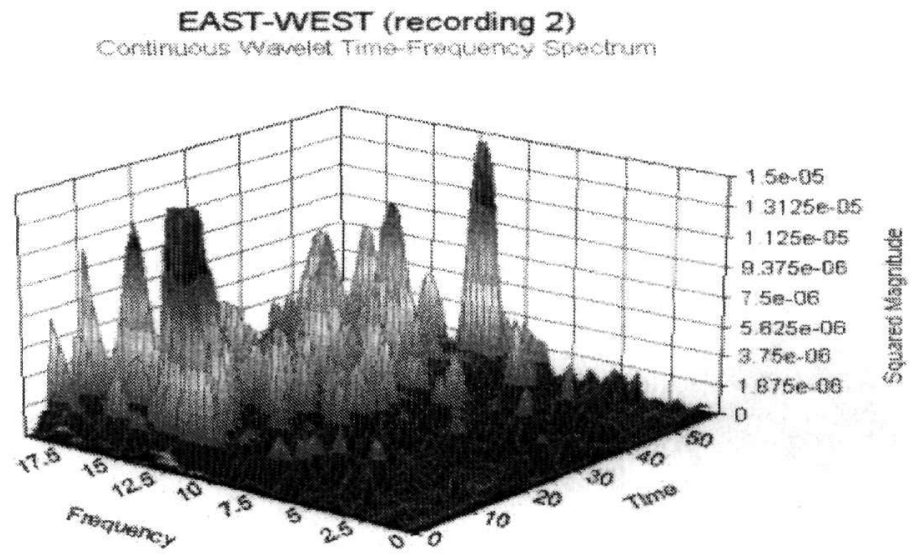

Figure 7a. Filtered wavelet spectrum for E-W component 


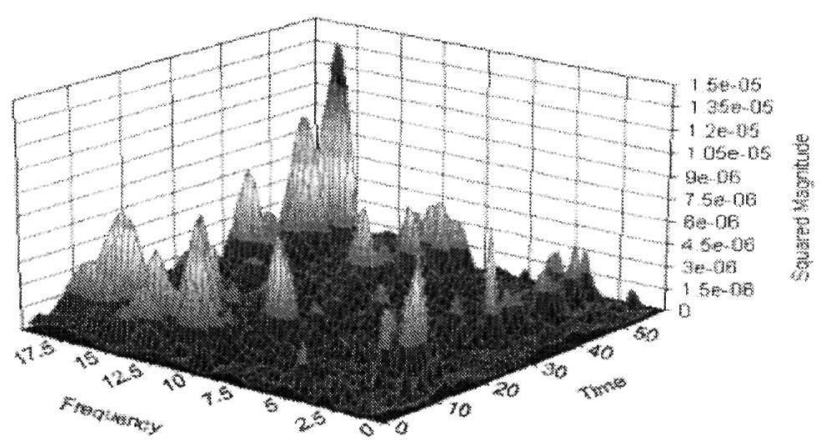

Figure 7b. Filtered wavelet spectrum for $\mathrm{N}-\mathrm{S}$ component VERTICAL (recording 2)

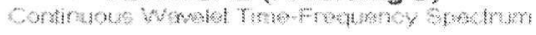

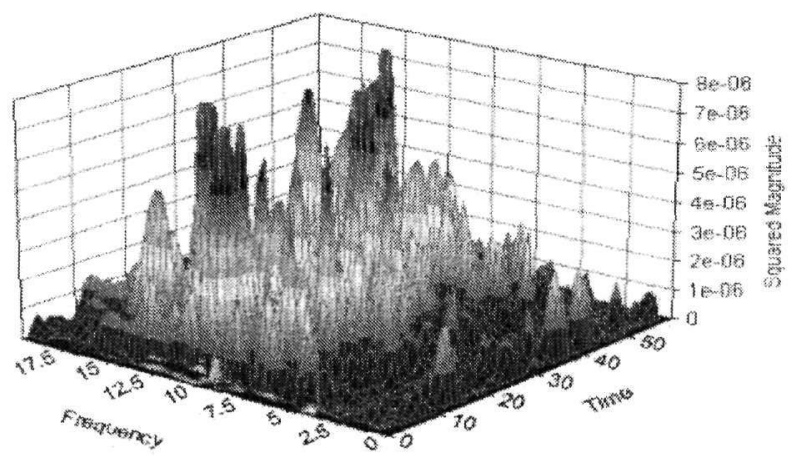

Figure 7c. Filtered wavelet spectrum for Vertical component

From the comparison of wavelet spectrums it is clear that the strong non-stationarities eliminated and at the same time lower amplitude components revealed.

After non-linear filtering we continue the estimation of HVSR to step c) and the result depicted in Figure 8.

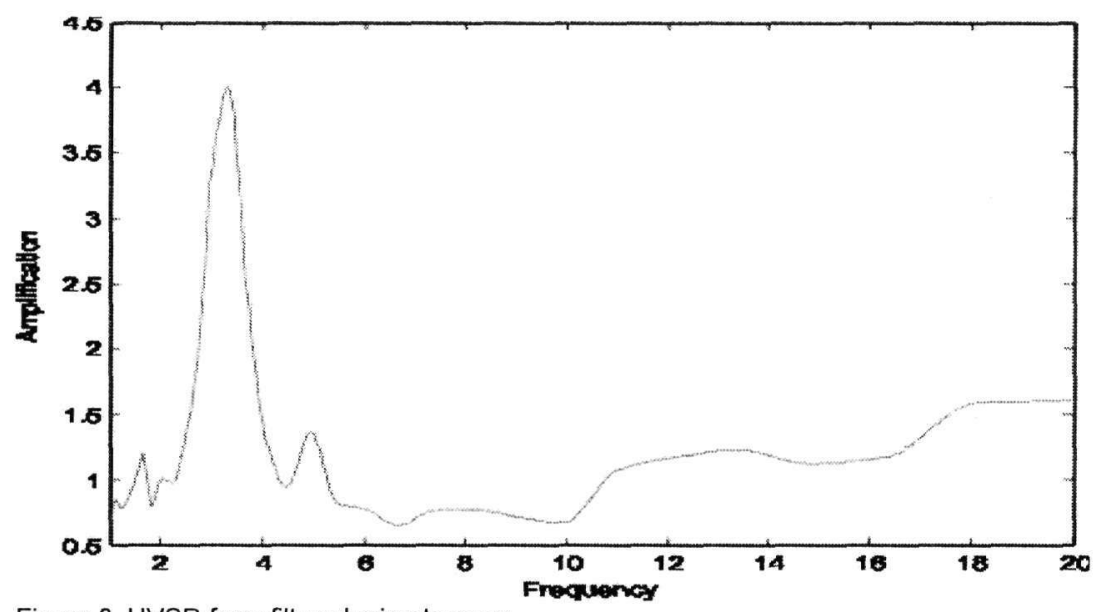

Figure 8. HVSR from filtered microtremors 
The latter estimation of HVSR indicates that the dominant frequency is around $3.5 \mathrm{~Hz}$ and not $16.4 \mathrm{~Hz}$ as we calculate using traditionally applied steps in HSVR technique. This result is expectable as long as we identify the non-stationarities with frequencies around $16 \mathrm{~Hz}$. After non-linear filtering the lower amplitude stationary components revealed and contribute to the spectral ratio resulting in a more accurate HVSR estimation.

\section{CONCLUSIONS}

The precision of the HVSR method for site effect investigations depends on the stationarity of the recorded microtremors. This is not obtainable when measurements take place in urban areas. In the present study we apply wavelet transform in order to identify non-stationarities in microtremors and then eliminate short period transients using non-linear wavelet filtering. This approach does not nullify the commonly used HVSR method but it is incorporated with it by adding one more procedure in order to achieve better and trustworthy results.

\section{ACKNOWLEDGEMENTS}

Research of the author G. Hloupis is funded by Greek National Foundation for Scholarships (IKY). The work of F.Vallianatos was supported by "ARCHIMIDIS" and CRINNO

\section{REFERENCES}

Chui, C.K., 1992. An introduction to wavelets, Academic press, New York

Konno, K, and T. Ohmachi, 1995. A smoothing function suitable for estimation of amplification factor of the surface ground from microtremors. J. JSCE $525,1-33$

Mallat, S.G., 1989. A theory for multiresolution signal decomposition : the wavelet representation, IEEE Transactions on Pattern Analysis and Machine Intelligence, 11, 674-693

Magri, L., Mucciarelli, M., Albarello, D.,1994. Estimates of site seismicity rates using ill-defined macroseismic data. Pure Appl. Geophys., 143(4),, 617-632

Mucciarelli, M., 1998, Reliability and applicability of Nakamura's technique using microtremors: an experimental approach, J Earthquake Eng, 2 (4), 625-638

Nakamura, Y., 1989. A method for dynamic characteristics estimation of subsurface using microtremor on the ground surface, QR of RTRI 30, 25-33

Nogoshi, M. and T. Igarashi, 1970. On the propagation characteristics of microtremors. J. Seism. Soc. Japan $23,264-280$

Nogoshi, M. and T. Igarashi, 1971. On the amplitude characteristics of the microtremors. J. Seism. Soc. Japan $24,24-40$

Odgen, R., 1997. Essential Wavelets for statistical applications and data analysis, Birkhauser, Boston

Papoulis, A., 1962. The Fourier integral and its applications. McGraw-Hill Press, New York 